\title{
Role of JNK activation in apoptosis: A double-edged sword
}

\author{
Jing LIU, Anning LIN* \\ Ben May Institute for Cancer Research, The University of Chicago, 5841 S. Maryland Avenue, MC 6027, Chicago, IL 60637, \\ USA
}

\begin{abstract}
JNK is a key regulator of many cellular events, including programmed cell death (apoptosis). In the absence of NF$\kappa \mathrm{B}$ activation, prolonged JNK activation contributes to TNF- $\alpha$ induced apoptosis. JNK is also essential for UV induced apoptosis. However, recent studies reveal that JNK can suppress apoptosis in IL-3-dependent hematopoietic cells via phosphorylation of the proapoptotic Bcl-2 family protein BAD. Thus, JNK has pro- or antiapoptotic functions, depending on cell type, nature of the death stimulus, duration of its activation and the activity of other signaling pathways.
\end{abstract}

Keywords: JNK, NF- $\kappa B$, apoptosis, TNF- $\alpha$, IL-3.

\section{INTRODUCTION}

Programmed cell death (apoptosis) plays a critical role in many biological processes in multicellular organisms, such as embryonic development, immune responses, tissue homeostasis and normal cell turnover [1-4]. Deregulation of apoptosis has been implicated in cancer $[2,4]$. The initiation and execution of apoptosis depend on activation of the extrinsic and/or intrinsic death pathways [5-8]. However, the apoptotic process is regulated by many intracellular signaling pathways, including the JNK pathway [9-12]. Understanding the molecular mechanisms by which JNK regulates apoptosis should provide insights into treatment and prevention of cancer.

c-Jun N-terminal protein kinase (JNK) is a subfamily of the mitogen activated protein kinase (MAPK) superfamily [13]. JNK has three isoforms (JNK1, 2 and 3), with slicing variant [9]. Among them, JNK1 and JNK2 are ubiquitously expressed while JNK3 is mainly expressed in neuronal and heart tissues [9-12]. JNK was originally identified by its ability to specifically phosphorylate the transcription factor c-Jun on its N-terminal transactivation domain at two serine residues, Ser63 and Ser73 [13]. Subsequent studies have shown that JNK also phosphorylates and regulates the activity of transcription factors other than c-Jun, including ATF2, Elk-1, p53 and c-Myc

*Correspondence: Anning LIN

Tel: +773-753-1408; Fax: +773-702-6260;

E-mail: alin@huggins.bsd.uchicago.edu
$[9,10,12,14]$ and non-transcription factors, such as members of the Bcl-2 family (Bcl-2, Bcl-xL Bim and BAD) [1517], in response to a variety of extracellular stimuli.

Activation of JNK is mediated by a MAP kinase module, i.e., MAP3K $\rightarrow$ MAP2K $\rightarrow$ MAPK [14], through sequential protein phosphorylation. So far, two MAP2Ks (JNKK1/ MKK4/SEK1 and JNKK2/MKK7) for JNK have been identified [18-22]. JNKKs are dual-specificity protein kinases, which phosphorylate JNK at Thr183 and Tyr185, leading to its activation. However, these two JNKKs appear to have different preferences for the phosphate-acceptor sites, with JNKK1 preferring Tyr185 [18, 23], and JNKK2 Thr183 [22-23]. Although phosphorylation of JNK at both Thr183 and Tyr 185 is required for its full activation, Tyr 185 phosphorylation seems to be a predominant one in TNF$\alpha$ induced JNK activation [22]. The biological significance of differential phosphorylation of JNK by JNKK1 and JNKK2 is not clear. Yet, it may allow JNK activation to be fine-toned in response to various stimuli. MAP3Ks for JNK include members of the MEKK family, ASK1, MLK, TAK1 and TPL-2 [12]. Genetic disruption of MEKK1 in mice abrogates JNK activation by certain stimuli [24-25]. However, the physiological relevance of other MAP3Ks in JNK activation has yet to be determined. JNK activity is also regulated by protein phosphatases, scaffold proteins such as JIP, $\beta$-arrestin and JSAP1, and NF- $\kappa \mathrm{B}$ [12].

The function and regulation of JNK has been the subject of several recent reviews [9-12]. This review will focus on the role of JNK in apoptosis, with emphasis on the molecular mechanisms by which JNK promotes or suppresses apoptosis. 
JNK AS A PROAPOPTOTIC PROTEIN KINASE

Numerous evidence shows that JNK can function as a proapoptotic kinase [9, 12]. Biochemical studies revealed that apoptosis induced by nerve growth factor (NGF) withdrawal in rat PC-12 pheochromocytoma cells was suppressed by inhibiting the JNK pathway, whereas activation of the JNK pathway by expressing a constitutively active MEKK1 [26] induced apoptosis in PC-12 cells [2728]. Genetic disruption experiments also showed that JNK has a proapoptotic role in apoptosis of neurons. In neuronal specific $J n k 3$ null mice, hippocampal neurons were resistant to apoptosis induced by kainate, an excitotoxic glutamate-receptor agonist [29] and isolated sympathetic neurons were resistant to NGF-withdrawal induced apoptosis [30]. In addition, mice deficient in both $j n k 1$ and jnk2 had decreased apoptosis in the hindbrain neuroepithelium at E9.25 during development [31-32]. Genetic disruption of jip 1 , which is a putative scaffold protein of the JNK pathway, also rendered hippocampal neurons resistant to kainate induced apoptosis [33]. It is likely that in neuronal apoptosis the proapoptotic function of JNK is mediate by c-Jun. Inhibition of c-Jun activity by neutralizing antibodies or a truncated mutant of c-Jun suppressed apoptosis induced by NGF withdrawal [34], whereas overexpression of c-Jun resulted in apoptosis in cultured sympathetic neurons [34]. Furthermore, sympathetic cJun $^{\text {AA63/73 }}$ neurons, in which endogenous c-Jun alleles were replaced with a non-phosphorylatable c-Jun(AA63/ 73) mutant, were resistant to apoptosis induced by kainite [35]. Yet, the molecular mechanism by which c-Jun mediates the proapoptotic function of JNK remains to be elucidated.

JNK is also involved in apoptosis induced by other death stimuli in non-neuronal cells $[9,10,12]$. Mouse embryonic fibroblasts (MEFs) deficient in both $j n k 1$ and $j n k 2$ were resistant to ultraviolet (UV) irradiation induced apoptosis [36]. Recent studies showed that genetic disruption of $J n k 1$ alleles alone was sufficient to render immortalized mouse fibroblasts resistant to UV induced apoptosis [37]. Whether c-Jun mediates the proapoptotic function of JNK in UV induced apoptosis in fibroblasts was controversial. It was proposed that the proapoptotic role of JNK in UV killing is independent of c-Jun-mediated transcription, since it was not affected by the protein synthesis inhibitor cycloheximide or the mRNA synthesis inhibitor actinomycin D [36]. However, MEFs isolated from c-Jun ${ }^{\mathrm{AA63} / 73} / \mathrm{c}-\mathrm{Jun}^{\mathrm{AA63/73}}$ mice were insensitive to UV-induced cell death [35], suggesting that c-Jun phosphorylation and activation is required for UV killing. Furthermore, it was reported that in response to UV, c-Jun inhibits p53-mediated cell cycle arrest, thereby promoting p53-mediated apoptosis [11]. Recent studies showed that c-Jun partially restored the apoptosis response of $J n k 1$ null fibroblasts when cells were exposed to low dose of UV but failed to do so when cells were irradiated with high dose of UV [37]. Since high dose of UV does not induce cell cycle arrest, c-Jun would not be able to regulate high dose of UV induced apoptosis [37]. Taken together, c-Jun transcription activity is likely required for apoptosis induced by low dose of UV but dispensable for apoptosis induced by high dose of UV.

Another example is the involvement of JNK in TNF- $\alpha$ induced apoptosis. TNF- $\alpha$ is a proinflammatory cytokine, which exerts its biological functions by activating multiple downstream signaling pathways, including caspases, IкB kinase (IKK) and JNK [12, 38, 40]. While activation of caspases is required for apoptosis, IKK activation inhibits apoptosis via the transcription factor NF- $\kappa$ B, whose target genes include inhibitors of apoptosis (IAPs) family [39-40]. Thus, TNF- $\alpha$ does not typically induce apoptosis unless NF- $\kappa$ B activation is inhibited [40]. The role of JNK activation in TNF- $\alpha$ induced apoptosis, however, was highly controversial, being suggested to have proapoptotic, antiapoptotic or no role in this process [41-43].

Recent studies have shown that in addition to inhibition of caspase activation, NF- $\kappa \mathrm{B}$ negatively regulates TNF- $\alpha$ induced JNK activation [12, 40, 44, 45, 46]. Activation of JNK by TNF- $\alpha$ was transient in wildtype fibroblasts, but was prolonged in cells deficient in either IKK, which is essential for NF- $\kappa \mathrm{B}$ activation by TNF- $\alpha$ [44] or RelA, which is a major transactivating subunit of NF- $\kappa B$ [44]. The molecular mechanism by which NF- $\kappa$ B inhibits JNK activation remains to be determined. Among the candidates of NF- $\kappa \mathrm{B}$-induced JNK inhibitors are X-chromosomelinked IAP (xiap) and gadd45 [40]. However, genetic disruption of either gene has no significant effect on JNK activation in vivo $[40,47,48]$, suggesting that a compensation mechanism may exist or the true inhibitor(s) is still at large. NF- $\kappa \mathrm{B}-$ mediated inhibition on JNK activation may target a unique component(s) in the TNF- $\alpha$ activated JNK pathway since it does not inhibit JNK activation by interleukin (IL)-1 [44] or UV (unpublished results). Since MAP2Ks (JNKK1/MKK4 and JNKK2/MKK7) are common components shared by TNF- $\alpha$, IL- 1 and UV signaling pathways, among which only TNF- $\alpha$ induced JNK activated is inhibited by NF- $\mathrm{KB}$, JNKK2/MKK 7 is unlikely targeted by NF- $\kappa \mathrm{B}-$ mediated inhibition. It is possible that $\mathrm{NF}-\kappa \mathrm{B}-\mathrm{mediated}$ inhibition targets a unique MAP3K in TNF- $\alpha$ signaling pathway (unpublished results).

Although the mechanism by which NF- $\kappa$ B inhibits JNK activation is still elusive, it is clear that NF- $\kappa \mathrm{B}-$ mediated inhibition on JNK activation is critical for cell survival [12, $40,44,45,46]$. In the absence of NF- $\kappa \mathrm{B}$ activation, JNK activation was prolonged in response to TNF- $\alpha[12,40$, $44,45,46]$. Inhibition of prolonged JNK activation by 
expressing JNKK2 (K149M), a dominant negative mutant of the JNK pathway [44] or using SP600125, a specific JNK inhibitor suppressed TNF- $\alpha$ induced apoptosis in RelA $^{-/-}$or $I k k^{-/-}$fibroblasts (unpublished results). Furthermore, TNF- $\alpha$ induced JNK activation was transient in TNF- $\alpha$ resistant human breast carcinoma MCF$7-\mathrm{R}$ cells, but was prolonged in TNF- $\alpha$ sensitive MCF-7 cells [45]. Restoration of transient JNK activation in MCF7 cells blocked TNF- $\alpha$ induced apoptosis [45].

\section{HOW JNK CONTRIBUTES TO APOPTOSIS}

It is clear that activation of the JNK pathway is involved in apoptosis induced by certain death stimuli, yet the underlying molecular mechanism is incompletely understood. Two hypotheses have recently been proposed to explain how JNK activation may contribute to apoptosis. The major difference between these two hypotheses is whether JNK activation is sufficient to induce or only promote apoptosis, as discussed below.

The key evidence that suggests JNK could induce apoptosis came from the observation that $j n k 1^{-/-j n k 2^{-/} \text {mice }}$ were resistant to apoptosis induced by UV irradiation, anisomycin and MMS [36]. Since UV was unable to induce cytochrome $\mathrm{C}$ release or depolarization of mitochondrial membrane potential in $j n k 1^{-/-} j n k 2^{-/-}$mice, it was proposed that JNK is an intrinsic component of the mitochondrial-dependent death pathway during stress-induced apoptosis [9]. In support of this conclusion, it was reported that in CHO cells the constitutively active JNKK2JNK1 fusion protein was sufficient to induce apoptosis by activating the intrinsic death pathway [49]. However, overexpression of a JNKK2-JNK1 fusion protein, which has constitutive JNK activity, did not induce apoptosis in RelA $^{-/-}$fibroblasts [44] or in other cells including wildtype or $I k k^{-/-}$fibroblasts and HeLa cells (unpublished results). In addition, no increased caspase activation or DNA fragmentation occurred in cells that express the constitutively active JNK when compared to cells that express control vector or a kinase-deficient JNKK2 (K149M)-JNK1 mutant (unpublished results). Identification of JNK target(s) in apoptosis is critical to test these two hypotheses. Recent studies suggested that JNK might induce apoptosis via modulating the proapoptotic Bcl-2 family protein BIM [50]. However, the physiological significance of the phosphorylation remains to be determined. Further studies are needed to determine whether JNK activation is sufficient to induce or only promote apoptosis.

Emerging evidence suggests that JNK may function as a modulator to "break the brake" on apoptosis, rather as a genetic component of the apoptotic machinery to induce apoptosis. The best example is the regulation of TNF-a induced apoptosis by JNK activation. As discussed earlier,
A
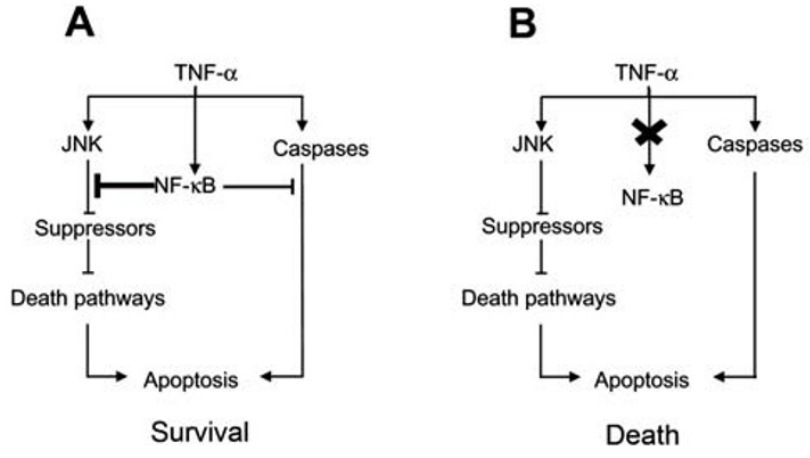

Fig. 1 The "breaking the brake" on apoptosis model that explains the role of JNK activation in TNF- $\alpha$ induced apoptosis. (A) While caspase activation initiates and executes apoptosis, prolonged JNK activation promotes apoptosis by inactivating suppressors of the mitochondrial-dependent death pathway, i.e., breaking the brake on apoptosis. Activation of NF- $\kappa$ B by TNF- $\alpha$ blocks caspase activation and prevents prolonged JNK activation, thereby inhibiting TNF$\alpha$ induced apoptosis. (B) Inactivation of NF- $\mathrm{KB}$ removes inhibition on both caspases and JNK, allowing TNF- $\alpha$ to induce apoptosis.

NF-kB suppresses TNF-a induced apoptosis by inhibiting caspases and preventing prolonged JNK activation [12, $40,45]$. In the absence of NF-kB activation, TNF- $\alpha$ induces apoptosis via activation of caspases and prolonged JNK activation [12, 40, 45]. However, prolonged JNK activation alone did not induce apoptosis, but was able to promote TNF- $\alpha$ induced apoptosis in the absence of NF$\kappa \mathrm{B}$ activation $[12,40,45]$. Recent studies have shown that only JNK 1 , but not JNK2, is activated by TNF- $\alpha$ and is required for TNF- $\alpha$ induced apoptosis in the absence of NF- $\kappa \mathrm{B}$ activation [37]. Not only was JNK2 activation by TNF- $\alpha$ negligible, JNK2 appeared to interfere with JNK1 activation by TNF- $\alpha$ [37]. Consistently, Jnk $2^{-/-}$fibroblasts were even more sensitive to TNF- $\alpha$ induced apoptosis [37]. Thus, JNK activation may contribute to apoptosis only if the apoptotic process has already been activated (Fig. 1). This idea is supported by a recent report that prolonged JNK activation resulted in the production of $\mathrm{jBID}$, which is a novel proteolytic fragment of BID [51]. It has been shown that jBID specifically induced the release of Smac from mitochondria and subsequently Smac displaced caspase 8 inhibitor c-IAP1 from TNF-receptor 1 complex, thereby allowing the initiation of apoptosis [51]. However, it is not clear how prolonged JNK activation results in proteolysis of BID and whether jBID is the effector downstream of prolonged JNK activation in vivo. It also remains to be determined whethere the model of "breaking the brake" on apoptosis can explain the proapoptotic role of JNK in apoptosis induced by other death insults. 


\section{JNK AS AN ANTI-APOPTOTIC PROTEIN KINASE}

JNK is not simply a proapoptotic protein kinase. JNK is activated by a variety of extracellular stimuli, only some of which induce apoptosis. When JNK does contribute to apoptosis, it is likely that it promotes, rather induces, apoptosis, as discussed above. Not surprisingly, evidence has been accumulating that suggests the involvement of JNK in cell survival or antiapoptosis. In mice deficient in both $j n k 1$ and jnk2, hindbrain [31] and forebrain regions [31-32] had enhanced apoptosis at E10.5. These observations suggest that JNK may be involved in cell survival in these brain regions during development, although the underlying molecular mechanism remains to be elucidated. JNK may be involved in T cell survival, since $J n k k 1^{-1-}$ immature thymocytes and peripheral mature $\mathrm{T}$ cells were highly sensitive to apoptosis mediated by Fas/CD95 and CD3 [52]. However, it has yet be determined whether the survival role of JNKK1 is mediated by $\mathrm{JNK}$ activation.

JNK may function as an anti-apoptotic protein kinase in certain tumors. Specific anti-sense oligonucleotides of JNK (JNKAS), which inhibits JNK activation, inhibited growth of certain types of tumor cells, probably by promoting apoptosis [53-54]. The anti-apoptotic function of JNK may be related to the status of p53 since JNKAS inhibited growth of certain p53-deficient, but not p53 positive, tumor cells [55]. This is likely that activation of the JNK pathway inhibits p53-induced cell cycle arrest and therefore promotes p53-induced apoptosis [11]. Thus, JNK may only exert its anti-apoptotic function in p53deficient tumor cells.

The antiapoptotic function of JNK in tumor cells suggests that JNK may be involved in tumorigenesis. Indeed, it has been reported that $\mathrm{JNK}$ functions as an antiapoptotic molecule in an isoform-specific manner in certain tumors. Tumor promoter 12-O-tetradecanolyphorbol-13-acetate (TPA)- induced skin papillomas were significantly suppressed in jnk2 null mice, due to enhanced apoptosis when compared to wild-type mice [56]. In contrast, TPAinduced skin tumors were augmented in jnk1 null mice [57]. It has been proposed that this is due to the unique stimulatory effect of JNK2 on AP-1 activity through activation of ERK, rather than c-Jun phosphorylation [57]. However, recent studies revealed that JNK1, but not JNK2, is activated by various extracellular stimuli, such as TNF$\alpha, \mathrm{UV}, \mathrm{IL}-1$ and TPA, and is required for TNF- $\alpha$ induced apoptosis [37]. Furthermore, JNK2 may interfere with JNK1 activity via its own "futile" phosphorylation [37]. The differential regulation of JNK1 and JNK2 by TPA may provide a molecular explanation about the distinct effects of JNK1 and JNK2 on TPA-induced skin papillomas.

\section{HOW JNK SUPPRESSES APOPTOSIS}

The molecular mechanism by which JNK suppresses apoptosis is incompletely understood. The best studied case about JNK-mediated cell survival so far is perhaps the involvement of JNK in suppression of apoptosis in pro-B lymphocytes [17]. In IL-3-dependent hematopoietic cells, IL-3 exerts its survival effects by inducing phosphorylation of the proapoptotic Bcl-2 family protein $\mathrm{BAD}$, triggering its sequestration by 14-3-3 in the cytoplasm [58]. This prevents inactivation of the pro-survival Bcl-2 family protein Bcl-xL by BAD [58] (Fig. 2). Previous studies showed that the survival role of IL-3 is mediated by Akt and mitochondria-membrane anchored PKA, which are responsible for phosphorylation of BAD at serine 112, 136 and 155 , thereby decreasing its association with Bcl-xL [59]. Recent studies showed that JNK also mediates the survival role of IL-3 [17]. JNK activity was suppressed by IL-3 withdrawal but activated by IL-3 re-addition [17]. IL-3 withdrawal induced apoptosis was promoted by inhibition of JNK but suppressed by a constitutively active JNK [17]. Unlike Akt and PKA that phosphorylate $\mathrm{BAD}$ at serine residues, JNK phosphorylated BAD at a novel Thr201 residue. Phosphorylation of BAD at Thr201 by JNK reduced its association with $\mathrm{Bcl}-\mathrm{xL}$ and replacement of Thr201 by aniline produced a BAD mutant that has enhanced proapoptotic activity [17]. In contrast to an early report [60], JNK does not phosphorylates BAD at Ser128 at all [17]. Thus, JNK functions as an antiapoptotic protein kinase in IL-3-dependent hematopoietic cells via phosphorylation and inactivation of the proapoptotic molecule Bad. The antiapoptotic function of JNK may provide a molecular mechanism by which JNK contributes to B lineage lymphoma/leukemia [17]. Future studies are needed to elucidate how JNK suppresses apoptosis induced by other death insults.

\section{CONCLUDING REMARKS}

The function of JNK in apoptosis is complex, as being suggested to have proapoptotic, antiapoptotic, or no role in the process. It is clear that JNK activation can contribute to apoptosis induced by certain death insults. Yet, it is debatable whether JNK is an intrinsic component of the apoptotic machinery or it only modulates apoptosis. JNK activation also leads to suppression of apoptosis induced by IL-3 withdrawal via phosphorylation and inactivation of the proapoptotic Bcl-2 family protein BAD. It is possible that JNK may suppress apoptosis induced by other death insults as well. It is most likely that JNK activation modulates the apoptotic process in a cell type and stimulus dependent manner. Since JNK deregulation has been implicated in cancer [9-12], investigation of the molecular mechanisms by which JNK regulates the apoptotic process should provide novel strategies to target the JNK 


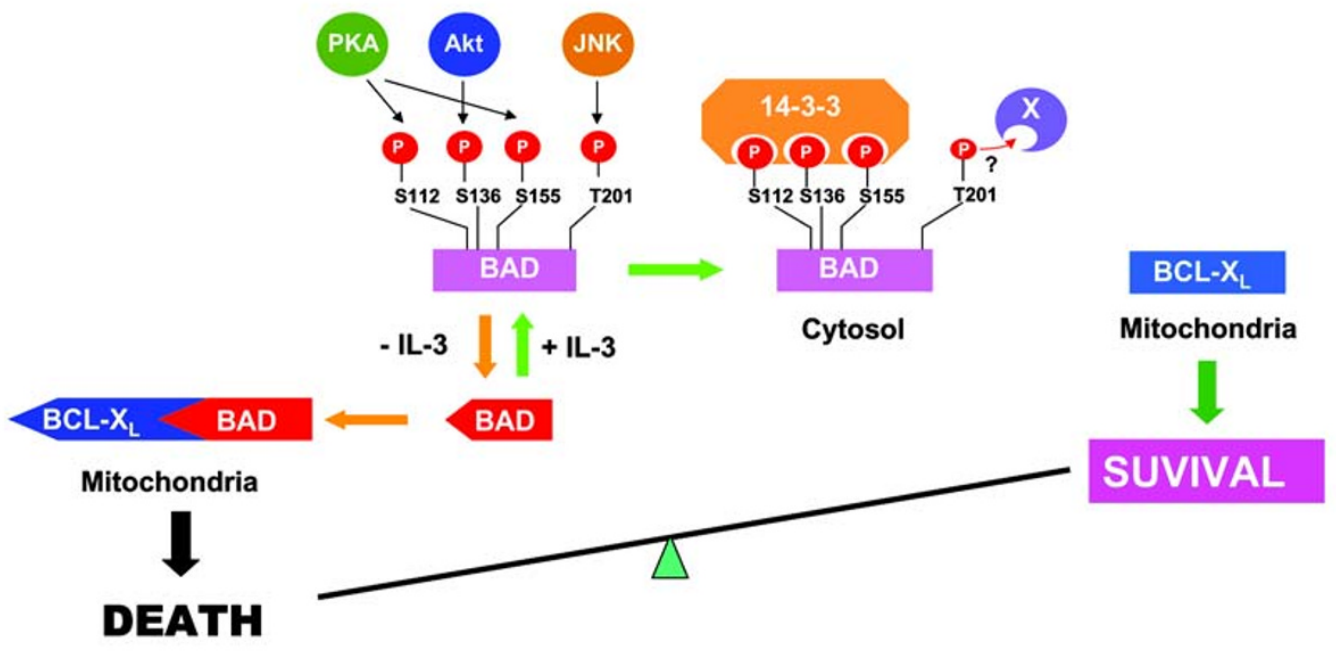

Fig. 2 JNK suppresses apoptosis in IL-3-depedent cells via phosphorylation of the pro-apoptotic Bcl-2 family protein BAD. In IL3-dependent hematopoietic cells, IL-3 activated JNK phosphorylates BAD at Thr201. The phosphorylation results in reduced association of BAD with $\mathrm{Bcl}-\mathrm{xL}$, thereby suppressing apoptosis.

pathway for prevention and treatment cancer.

\section{ACKNOWLEDGEMENTS}

We thank members of the Lin laboratory for helpful discussion. We apologize to those authors for not being able to directly cite their work due to space constraints. This work is supported in part by grants from the National Institutes of Health (NIH).

\section{REFERENCES}

1 Kerr JF, Wyllie AH, Currie AR. Apoptosis: a basic biological phenomenon with wide-ranging implications in tissue kinetics. Br J Cancer 1972; 26:239-57.

2 Rinkenberger JL, Korsmeyer SJ. Errors of homeostasis and deregulated apoptosis. Curr Opin Genet Dev 1997; 7:589-96.

3 Jacobson MD, Weil M, Raff MC. Programmed cell death in animal development. Cell 1997; 88:347-54.

4 Thompson CB. Apoptosis in the pathogenesis and treatment of disease. Science 1995; 267:1456-62.

5 Ashkenazi A, Dixit VM. Death receptors: signaling and modulation. Science 1998; 281:1305-1308.

6 Cohen GM. Caspases: the executioners of apoptosis. Biochem J 1997; 326:1-16.

7 Green DR, Reed JC. Mitochondria and apoptosis. Science 1998; 281:1309-12.

8 Thornberry NA, Lazebnik Y. Caspases: enemies within. Science 1998; 281:1312-6.

9 Davis RJ. Signal transduction by the JNK group of MAP kinases. Cell 2000; 103: 239-52.

10 Chang L, Karin M. Mammalian MAP kinase signalling cascades.
Nature $2001 ;$ 410: 37-40.

11 Shaulian E, Karin M. AP-1 as a regulator of cell life and death. Nat Cell Biol 2002; 4:E131-6.

12 Lin A. Activation of the JNK signaling pathway: breaking the break on apoptosis. Bioessays 2003; 25:1-8.

13 Hibi M, Lin A, Smeal T, Minden A, Karin M. Identification of an oncoprotein- and UV-responsive protein kinase that binds and potentiates the c-Jun activation domain. Genes Dev 1993; 7: 2135-48

14 Karin M. The regulation of AP-1 activity by mitogen-activated protein kinases. J Biol Chem 1995; 270:16483-6.

15 Yamamoto K, Ichijo H, Korsmeyer SJ. BCL-2 is phosphorylated and inactivated by an ASK1/Jun N-terminal protein kinase pathway normally activated at G(2)/M. Mol Cell Biol 1999; 19: 8469-78.

16 Maundrell K, Antonsson B, Magnenat E, et al. Bcl-2 undergoes phosphorylation by c-Jun N-terminal kinase/stress- activated protein kinases in the presence of the constitutively active GTPbinding protein Rac1. J Biol Chem 1997; 272:25238-42.

$17 \mathrm{Yu} \mathrm{C}$, Minemoto Y, Zhang J, et al. JNK suppresses apoptosis via phosphorylation of the proapoptotic Bcl-2 family protein BAD. Molecular Cell 2004, 13:329-40.

18 Lin A, Minden A, Martinetto H, et al. Identification of a dual specificity kinase that activates the Jun kinases and p38-Mpk2. Science 1995; 268:286-90.

19 Derijard B, Raingeaud J, Barrett T, et al. Independent human MAP-kinase signal transduction pathways defined by MEK and MKK isoforms. Science 1995; 267:682-5.

20 Sanchez I, Hughes RT, Mayer BJ, et al. Role of SAPK/ERK kinase-1 in the stress-activated pathway regulating transcription factor c-Jun. Nature 1994; 372:794-8.

$21 \mathrm{Lu} \mathrm{X}$, Nemoto S, Lin A. Identification of c-Jun NH2-terminal 
protein kinase (JNK)-activating kinase 2 as an activator of JNK but not p38. J Biol Chem 1997; 272:24751-4.

22 Tournier C, Dong C, Turner TK, et al. MKK7 is an essential component of the JNK signal transduction pathway activated by proinflammatory cytokines. Genes Dev 2001; 15:1419-26.

23 Fleming Y, Armstrong CG, Morrice N, et al. Synergistic activation of stress-activated protein kinase $1 / \mathrm{c}$-Jun $\mathrm{N}$-terminal kinase (SAPK1/JNK) isoforms by mitogen-activated protein kinase kinase 4 (MKK4) and MKK7. Biochem J 2000; 352 Pt 1:145-4.

$24 \mathrm{Xia} \mathrm{Y,} \mathrm{Makris} \mathrm{C,} \mathrm{Su} \mathrm{B,} \mathrm{et} \mathrm{al.} \mathrm{MEK} \mathrm{kinase} 1$ is critically required for c-Jun $\mathrm{N}$-terminal kinase activation by proinflammatory stimuli and growth factor-induced cell migration. Proc Natl Acad Sci U S A 2000; 97:5243-8.

25 Yujiri T, Sather S, Fanger GR, Johnson GL. Role of MEKK1 in cell survival and activation of JNK and ERK pathways defined by targeted gene disruption. Science 1998; 282:1911-14.

26 Minden A, Lin A, McMahon M, et al. Differential activation of ERK and JNK mitogen-activated protein kinases by Raf-1 and MEKK. Science 1994; 266:1719-23.

27 Xia Z, Dickens M, Raingeaud J, Davis RJ, Greenberg ME. Opposing effects of ERK and JNK-p38 MAP kinases on apoptosis. Science 1995; 270:1326-31.

28 Le-Niculescu H, Bonfoco E, Kasuya Y, et al. Withdrawal of survival factors results in activation of the JNK pathway in neuronal cells leading to Fas ligand induction and cell death. Mol Cell Biol 1999; 19:751-63.

29 Yang DD, Kuan CY, Whitmarsh AJ, et al. Absence of excitotoxicity-induced apoptosis in the hippocampus of mice lacking the jnk3 gene. Nature 1997; 389:865-70.

30 Bruckner SR, Tammariello SP, Kuan CY, et al. JNK3 contributes to c-Jun activation and apoptosis but not oxidative stress in nerve growth factor-deprived sympathetic neurons. J Neurochem 2001; 78:298-303.

31 Sabapathy K, Jochum W, Hochedlinger K, et al. Defective neural tube morphogenesis and altered apoptosis in the absence of both JNK1 and JNK2. Mech Dev 1999; 89:115-24.

32 Kuan CY, Yang D, Samanta Roy DR, et al. The Jnk1 and Jnk2 protein kinases are required for regional specific apoptosis during early brain development. Neuron 1999; 22:667-76.

33 Whitmarsh AJ, Kuan CY, Kennedy NJ, et al. Requirement of the JIP1 scaffold protein for stress-induced JNK activation. Genes Dev 2001; 15:2421-32.

34 Ham J, Babij C, Whitfield J, et al. A c-Jun dominant negative mutant protects sympathetic neurons against programmed cell death. Neuron 1995; 14:927-39.

35 Behrens A, Sibilia M, Wagner EF. Amino-terminal phosphorylation of c-Jun regulates stress-induced apoptosis and cellular proliferation. Nat Genet 1999; 21:326-9.

36 Tournier C, Hess P, Yang DD, et al. Requirement of JNK for stress-induced activation of the cytochrome c-mediated death pathway. Science 2000; 288:870-4.

37 Liu J, Minemoto Y, Lin A. c-Jun N-terminal protein kinase 1 (JNK1), but not JNK2, is essential for tumor necrosis factor alpha-induced c-Jun kinase activation and apoptosis. Mol Cell Biol 2004; 24:10844-56.

38 Baud V, Karin M. Signal transduction by tumor necrosis factor and its relatives. Trends Cell Biol 2001; 11:373-7.

39 Baldwin AS. Control of oncogenesis and cancer therapy resistance by the transcription factor NF- $\mathrm{BB}$. J Clin Invest 2001;
107:241-6

40 Karin M, Lin A. NF- $\kappa$ B at the crossroads of life and death. Nat Immunol 2002; 3:221-7.

41 Liu ZG, Hsu H, Goeddel DV, Karin M. Dissection of TNF receptor 1 effector functions: JNK activation is not linked to apoptosis while NF- $\mathrm{KB}$ activation prevents cell death. Cell 1996; 87:565-76.

42 Verheij M, Bose R, Lin XH, et al. Requirement for ceramideinitiated SAPK/JNK signalling in stress-induced apoptosis. Nature 1996; 380:75-9.

43 Natoli G, Costanzo A, Ianni A, et al. Activation of SAPK/JNK by TNF receptor 1 through a noncytotoxic TRAF2- dependent pathway. Science 1997; 275:200-3.

44 Tang G, Minemoto Y, Dibling B, et al. Inhibition of JNK activation through NF-kB target genes. Nature 2001; 414:313-7.

45 Tang F, Tang G, Xiang J, et al. Absence of NF- $\kappa$ B-mediated inhibition of c-Jun N-terminal kinase activation contributes to tumor necrosis factor a induced apoptosis. Mol Cell Biol 2002, 22:8571-9.

46 Maeda S, Chang L, Li ZW, et al. IKKbeta is required for prevention of apoptosis mediated by cell-bound but not by circulating TNF- $\alpha$. Immunity 2003, 19:725-37.

47 Harlin H, Reffey SB, Duckett CS, Lindsten T, Thompson CB. Characterization of XIAP-deficient mice. Mol Cell Biol 2001; 21:3604-8.

48 Amanullah A, Azam N, Balliet A, et al. Cell survival and a Gadd45factor deficiency. Nature 2003; 424:741-2.

49 Lei K, Nimnual A, Zong WX, et al. The Bax subfamily of Bcl2related proteins is essential for apoptotic signal transduction by c-Jun NH(2)-terminal kinase. Mol Cell Biol 2002; 22:4929-42.

50 Lei K, Davis RJ. JNK phosphorylation of Bim-related members of the Bcl2 family induces Bax-dependent apoptosis. Proc Natl Acad Sci U S A. 2003; 100:2432-7.

51 Deng Y, Ren X, Yang L, Lin Y, Wu X. A JNK-dependent pathway is required for TNF- $\alpha$ induced apoptosis. Cell 2003; 115:61-70.

52 Nishina H, Fischer KD, Radvanyi L, et al. Stress-signalling kinase Sek1 protects thymocytes from apoptosis mediated by CD95 and CD3. Nature 1997; 385:350-3.

53 Bost F, McKay R, Bost M, et al. The Jun kinase 2 isoform is preferentially required for epidermal growth factor-induced transformation of human A549 lung carcinoma cells. Mol Cell Biol 1999; 19:1938-49.

54 Potapova O, Anisimov SV, Gorospe M, et al. Targets of c-Jun $\mathrm{NH}(2)$-terminal kinase 2-mediated tumor growth regulation revealed by serial analysis of gene expression. Cancer Res 2002; 62:3257-63.

55 Potapova O, Gorospe M, Dougherty RH, et al. Inhibition of cJun N-terminal kinase 2 expression suppresses growth and induces apoptosis of human tumor cells in a p53-dependent manner. Mol Cell Biol 2000; 20:1713-22.

56 Chen N, Nomura M, She QB, et al. Suppression of skin tumorigenesis in c-Jun NH(2)-terminal kinase-2- deficient mice. Cancer Res 2001; 61:3908-12.

57 She QB, Chen N, Bode AM, Flavell RA, Dong Z. Deficiency of c-Jun-NH(2)-terminal kinase-1 in mice enhances skin tumor development by $12-\mathrm{O}$-tetradecanoylphorbol-13-acetate. Cancer Res 2002; 62:1343-8.

58 Zha J, Harada H, Yang E, Jockel J, Korsmeyer SJ. Serine phosphorylation of death agonist BAD in response to survival factor 


\section{Role of JNK in apoptosis}

results in binding to 14-3-3 not BCL-X(L). Cell 1996; 87:619-28.

59 Gross A, McDonnell JM, Korsmeyer SJ. BCL-2 family members and the mitochondria in apoptosis. Genes Dev. 1999; 13: 1899-911.
60 Donovan N, Becker EB, Konishi Y, Bonni A. JNK phosphorylation and activation of BAD couples the stress-activated signaling pathway to the cell death machinery. J Biol Chem 2002; 277: 40944-9. 